\title{
COMPORTAMENTO TERMODINÂNICO DE GRÃOS DE UVA DURANTE O PROCESSO DE SECAGEM
}

\author{
G. JOHANN ${ }^{1 *}$, N.C. PEREIRA ${ }^{1}$, E.A. SILVA ${ }^{2}$ \\ ${ }^{1}$ Universidade Estadual de Maringá, Departamento de Engenharia Química \\ ${ }^{2}$ Universidade Estadual do Oeste do Paraná, Curso de Engenharia Química \\ "grajohann@yahoo.com.br
}

\begin{abstract}
RESUMO
Foram determinadas as funções termodinâmicas diferenciais e integrais (entalpia e entropia) e o calor líquido isostérico de dessorção de grãos de uva cultivar Vitis vinífera. O calor total de dessorção calculado variou de 4864 a $2591 \mathrm{~kJ} / \mathrm{kg}$, análogo a dados da literatura, sendo que a quantidade de energia requerida para a dessorção aumentou com a redução do conteúdo de umidade. A entropia diferencial caiu drasticamente com o aumento da umidade, conforme literatura, apresentando valor máximo de 5,34 e mínimo de $0,77 \mathrm{~J} / \mathrm{mol} \mathrm{K}$. A pressão de espalhamento, analisada nas temperaturas de $25,35 \mathrm{e}$ $50{ }^{\circ} \mathrm{C}$, aumentou discretamente com o aumento da atividade da água, sendo que com o aumento da temperatura, os valores foram reduzidos, a mesma tendência foi observada na literatura para outras sementes. A entalpia integral permaneceu aproximadamente constante, $220 \mathrm{~kJ} / \mathrm{mol}$, sofrendo decréscimo acentuado a partir de 0,05 (b.s.), valores próximos aos da literatura. A entropia integral aumentou com o acréscimo do teor de umidade, variando ente aproximadamente $-0,702$ a $0,013 \mathrm{~kJ} / \mathrm{mol} \mathrm{K}$, entre $0,01 \mathrm{e}$ 0,07 (b.s.).
\end{abstract}

\section{INTRODUÇÃO}

Uvas são uma das mais populares e saborosas frutas do mundo, sendo que a maior parte de sua produção é usada para fabricação de vinhos (FADHEL et al. 2005), gerando como principais resíduos o pendão, bagaço, lodo e sementes (BUSTAMANTE et al. 2008). As sementes de uva são ricas em ácidos graxos insaturados, que podem representar até cerca de $89 \%$ da composição total dos óleos (DAVIDOV-PARDO e MCCLEMENTS, 2015). A extração do óleo envolve diversas operações preliminares, como limpeza, descasque, secagem e moagem, sendo a secagem a etapa que mais consome energia.
Diferentes métodos de secagem são usados na secagem de frutas e vegetais, sendo que a aplicação de ar aquecido é o método mais extensamente empregados nas diversas tecnologias pós-colheita de produtos agrícolas (GUINÉ, HENRIQUES e BARROCA, 2012; STURM, VEGA e HOFACKER, 2014), porém, pode consumir até $15 \%$ de toda a energia utilizada no processo produtivo (PERUSSELLO et al. 2014).

Mais de 70 milhões de toneladas de água são removidas de grãos anualmente, para garantir o armazenamento adequado dos mesmos, isso é equivalente a evaporar um cubo de água com mais de $400 \mathrm{~m}$ de comprimento lateral (RAGHAVAN e SOSLE, 2007). Para Alonso (2011), o consumo de 
energia na secagem de grãos é essencialmente empregado em três processos: aquecimento do grão, evaporação da água e aquecimento do ar de secagem, dessa forma, para que o processo seja eficiente, é necessário recuperar energia de um desses três processos, entretanto, o desenvolvimento dessas alternativas tem levado muitas décadas. De acordo Verna (1994), somente os Estados Unidos consomem 15 milhões de barris de petróleo bruto por ano na secagem de grãos, tornando a secagem uma das operações de importante fonte de consumo de energia.

De acordo com Fluck e Baird (1980), a energia requerida na secagem de grãos, em condições ideais, varia de 2500 a $2670 \mathrm{~kJ} / \mathrm{kg}$, dependendo da temperatura, embora para Gunasekaran e Thompson (1986), valores da ordem de 3000 a $8000 \mathrm{~kJ} / \mathrm{kg}$ sejam atualmente necessários. Esses dados indicam a eminente necessidade de otimizar os processos de secagem de grãos, sendo que o primeiro passo para tal é o conhecimento do comportamento termodinâmico envolvido na operação de secagem.

Os objetivos do presente trabalho são determinar as funções termodinâmicas diferenciais e integrais (entalpia e entropia), a partir das isotermas de dessorção de grãos de uva Vitis vinifera.

\section{MATERIAIS E MÉTODOS}

\subsection{Calor isostérico de dessorção, entalpia e entropia diferenciais de dessorção}

O calor líquido isostérico de dessorção, $\lambda_{\mathrm{N}}(\mathrm{kJ} / \mathrm{kg})$, é definido como o calor total de dessorção, $\lambda_{\mathrm{D}}(\mathrm{kJ} / \mathrm{kg})$ menos o calor latente de vaporização da água pura na temperatura do sistema, $\lambda_{\mathrm{V}} \quad(\mathrm{kJ} / \mathrm{kg}), \quad$ Equação 1 , é frequentemente avaliado a partir de dados de equilíbrio em diferentes temperaturas (TOLABA et al. 2004).

$\lambda_{\mathrm{N}}=\lambda_{\mathrm{D}}-\lambda_{\mathrm{V}}$
Ele é uma medida da força de ligação do grão em água (MCMINN e MAGEE, 2003), sendo geralmente influenciado pelos métodos de secagem (PEDRO, TELIS-ROMERO e TELIS, 2010), e é considerado um parâmetro essencial nos cálculos da energia requerida para os processos de secagem (IGUAZ e VÍRSEDA, 2007).

O calor de vaporização da água pura, conforme Treybal (1980), é definido pela Equação 2, em função da temperatura, $\mathrm{T}\left({ }^{\circ} \mathrm{C}\right)$.

$\lambda_{\mathrm{V}}=2.162 \times 10^{3}+2 \mathrm{~T}$

Existem diversos métodos para se obter o calor de dessorção nos processos de secagem, sendo que o método mais extensamente usado é a aplicação da equação de Clausius-Clapeyron às isotermas de dessorção, relacionando a mudança da atividade de água com a temperatura (BECKER e SALLANS, 1956). Um método alternativo foi desenvolvido por (OTHMER, 1940), que assume o calor de dessorção e condensação como tendo a mesma dependência funcional com a temperatura, esta consideração é menos restritiva que a do método de Clapeyron (SILVA et al. 2002).

Close e Banks (1972) obtiveram a Equação de Othmer, Equação 3, para a entalpia diferencial, assumindo que o processo de dessorção é termodinamicamente reversível, sendo que o vapor se comporta como gás ideal e que os efeitos de superfície são negligenciáveis.

$\left(\frac{\partial \ln \mathrm{P}_{\mathrm{V}}}{\partial \ln \mathrm{P}_{\mathrm{P}}}\right)_{\mathrm{X}_{\mathrm{E}}}=\frac{\lambda_{\mathrm{D}}}{\lambda_{\mathrm{V}}}$

A Equação de Othmer estabelece que, uma vez conhecida a umidade de equilíbrio, $\mathrm{X}_{\mathrm{E}}$, em base seca (b.s.), em diferentes temperaturas e pressões de vapor, $\mathrm{P}_{\mathrm{V}}(\mathrm{Pa})$, e em valores conhecidos de pressões parciais, $\mathrm{P}_{\mathrm{P}}$ $(\mathrm{Pa})$, a relação entre o calor de vaporização da 
água pura e o calor total de dessorção pode ser determinada.

Os dados experimentais utilizados no presente estudo foram obtidos por Menezes (2014), que utilizou o método dinâmico, estabelecendo que a isoterma que melhor representou o processo de secagem dos grãos de uva Vitis vinifera foi o Modelo de Henderson (1952), conforme Equação 4, que relaciona a umidade de equilíbrio com a atividade da água, $\mathrm{a}_{\mathrm{w}}(-)$. A Tabela 1 , obtida por Menezes (2014), elenca os valores dos parâmetros da isoterma de equilíbrio, B e D.

$X_{E}=D\left[\frac{1}{T} \ln \left(\frac{1}{1-a_{w}}\right)\right]^{B}$

Tabela 1 - Parâmetros da isoterma de dessorção de Henderson (1952) para grãos de uva cultivar Cabernet

\begin{tabular}{ccc}
\hline $\mathrm{T}\left({ }^{\circ} \mathrm{C}\right)$ & $\mathrm{B}$ & $\mathrm{D}$ \\
\hline 25 & 0,6401 & 0,7447 \\
35 & 0,7093 & 1,1315 \\
50 & 0,9838 & 3,0280
\end{tabular}

Fonte: Menezes (2014)

O calor de dessorção foi calculado na faixa de umidade de equilíbrio entre 0,01 e 0,1 (b.s.), a partir das curvas dos logaritmos naturais da pressão parcial e da pressão de vapor, a partir da equação com melhor ajuste em relação à temperatura. Os cálculos foram realizados no software Statistica $7^{\circledR}$.

De acordo com Togrul e Arslan (2006), a entropia diferencial, ou entropia de dessorção, $\mathrm{S}_{\mathrm{D}}(\mathrm{kJ} / \mathrm{kg} \mathrm{K})$, de determinado material é proporcional ao número de sítios de sorção disponíveis a um nível específico de energia. A entropia diferencial de dessorção, para cada conteúdo de umidade, foi determinada a partir da Equação 5, com melhor ajuste em relação aos dados de equilíbrio, conforme (MADAMHA, DRISCOLL e
BUCKLE, 1996), em que $\mathrm{R}$ é a constante dos gases ideais, $(8,314 \mathrm{~J} / \mathrm{mol} \mathrm{K})$.

$\left(-\ln \mathrm{a}_{\mathrm{W}}\right)_{\mathrm{X}_{\mathrm{E}}}=\frac{\lambda_{\mathrm{D}}}{\mathrm{RT}}-\frac{\mathrm{s}_{\mathrm{D}}}{\mathrm{R}}$

\subsection{Entalpia e entropia integrais líquidas}

A entalpia integral líquida, $\mathrm{H}_{\mathrm{I}}(\mathrm{kJ} / \mathrm{kg})$ representa a energia total necessária para a realização de determinado trabalho, indicando a força de ligação das moléculas de água e o grão (GAL, 1975). Ela é calculada de forma similar ao cálculo do calor de dessorção, fazendo uso de uma constante chamada de spreading pressure, ou pressão de espalhamento, $\phi\left(\mathrm{J} / \mathrm{m}^{2}\right)$, em lugar da umidade de equilíbrio (BENADO e RIZVI, 1985). A equação de Clausius-Clapeyron com a pressão de espalhamento constante é descrita pela Equação 6.

$\left(\frac{\partial \ln \mathrm{a}_{\mathrm{W}}}{\partial(1 / \mathrm{T})}\right)_{\phi}=-\frac{\mathrm{H}_{\mathrm{I}}}{\mathrm{R}}$

A entalpia integral líquida é o coeficiente angular da relação entre o logaritmo natural da atividade da água e o inverso da temperatura à pressão de espalhamento constante, que, de acordo com Stamm (1964), é a energia livre da superfície de sorção, podendo ser estimada pela da diferença entre o potencial de superfície dos sítios de sorção livres e das moléculas sorvida. O cálculo da pressão de espalhamento foi realizado conforme procedimento analítico descrito por Iglesias (1976) e Fasina, Ajibola e Tyler (1999), Equação 7 , em que $K_{B}$ é a constante de Boltzmann's, $1.38 \times 10^{-23} \mathrm{~J} / \mathrm{K}, \mathrm{A}_{\mathrm{m}}$ é a área da molécula de água, $1.06 \times 10^{-19} \mathrm{~m}^{2}$ e $\mathrm{X}_{\mathrm{m}}$ é a umidade da monocamada (b.s.)

$\phi=\frac{K_{B} T}{A_{m}} \int_{0}^{a_{w}} \frac{X_{E}}{x_{m} a_{w}} d a_{w}$

Para a determinação da pressão de espalhamento, uma relação empírica entre a atividade da água e a umidade de equilíbrio 
deve ser usada. No presente trabalho foi empregada a solução proposta por Dent (1977), fornecendo a forma final expressa pela Equação 8, Ouertani et al. (2014).

$\phi=\frac{\mathrm{K}_{\mathrm{B}} \mathrm{T}}{\mathrm{A}_{\mathrm{m}}} \ln \left[\frac{1+\mathrm{A}-\mathrm{Ba}_{\mathrm{w}}}{1-\mathrm{Ba}_{\mathrm{w}}}\right]$

A equação de BET (BRUNAUER et al. 1938), Equação 9, foi usada determinação dos parâmetros A e B da Equação 8.

$X_{E}=\frac{A B a_{w}}{\left(1-a_{w}\right)\left[1+(A+1) a_{w}\right]}$

Por sua vez, a entropia integral líquida, $\mathrm{S}_{\mathrm{I}}(\mathrm{kJ} / \mathrm{mol} \mathrm{K})$, descreve o grau de desordem e aleatoriedade do movimento da umidade, e é expressa pela Equação 10, (MAZZA e LE MAGUER, 1978).

$\mathrm{S}_{\mathrm{I}}=-\frac{\mathrm{H}_{\mathrm{I}}}{\mathrm{T}}-\mathrm{R} \ln \mathrm{a}_{\mathrm{w}}{ }^{*}$

Em que $\mathrm{a}_{\mathrm{w}}{ }^{*}$ é a média geométrica de $\mathrm{a}_{\mathrm{w}}$, nas três temperaturas estudadas, calculada à pressão de espalhamento constante, conforme Equação 11.

$\mathrm{a}_{\mathrm{w}}{ }^{*}=\left(\prod_{\mathrm{n}=1}^{3} \mathrm{a}_{\mathrm{w}}\right)^{1 / \mathrm{n}}$

\section{RESULTADOS E DISCUSSÃO}

3.1 Calor líquido isostérico de dessorção, entalpia e entropia diferenciais de dessorção

Foram calculadas as pressões parciais e de vapor, nas temperaturas da Tabela 1, sendo que na Figura 1 é possível constatar relação linear entre $\ln \mathrm{P}_{\mathrm{V}}$ e $\ln \mathrm{P}_{\mathrm{P}}$. As inclinações dos pontos calculados para os seis valores de umidade de equilíbrio foram determinadas por meio de regressão linear Os valores de $R^{2}$ ficaram entre 0,989 e 0,998, conforme Tabela 2.

Os dados dos coeficientes angulares, $\lambda_{\mathrm{D}} / \lambda_{\mathrm{V}}$, foram correlacionados conforme a Equação 12, por meio do Software Statistica
$7^{\circledR}$, apresentado uma correlação de 0,999 , evidenciando precisão no uso da equação de Othmer.

$\lambda_{\mathrm{D}} / \lambda_{\mathrm{V}}=1,0692+$

$1,4518 \exp \left(-29,2897 \mathrm{X}_{\mathrm{E}}\right)$

Figura 1 - Relação entre $\ln P_{V}$ e $\ln P_{P}$

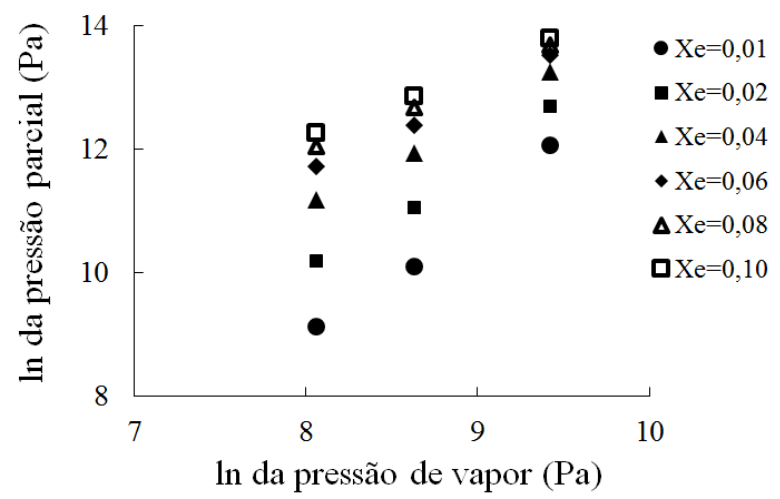

Tabela 2 - Valores da razão estimada do calor de dessorção pelo calor de vaporização da água

\begin{tabular}{ccc}
\hline $\mathrm{X}_{\mathrm{E}}$ & $\lambda_{\mathrm{D}} / \lambda_{\mathrm{V}}$ & $\mathrm{R}^{2}$ \\
\hline 0,010 & 2,167 & 0,989 \\
0,020 & 1,851 & 0,992 \\
0,040 & 1,523 & 0,995 \\
0,060 & 1,336 & 0,996 \\
0,080 & 1,214 & 0,998 \\
0,100 & 1,133 & 0,998
\end{tabular}

Os valores do calor de dessorção do processo de secagem de grãos de uva da variedade Cabernet Sauvignon, obtidos pela Equação de Othmer em função da umidade de equilíbrio, foram calculados pela Equação 12, e são ilustradas na Figura 2, que apresenta o comportamento do calor total de dessorção, calor líquido de dessorção e de vaporização, durante o processo de secagem, estes últimos foram calculados pelas Equações 1 e 2, respectivamente.

É possível verificar na Figura 2, que com o aumento do conteúdo de umidade o calor total de dessorção aproxima-se do calor de vaporização da água na mesma temperatura, pois quanto mais água está presente no material, a energia de ligação entre as moléculas é enfraquecida aproximando-se das 
moléculas de água em estado líquido. Quando o material apresenta um menor conteúdo de umidade, a energia de interação entre as moléculas de água e a primeira camada de sorção do sólido é maior do que a energia que mantém as moléculas unidas nas sucessivas camadas.

Figura 2 - Calores total e líquido de dessorção e calor de vaporização (na temperatura de $373,15 \mathrm{~K}$, equivalente a $2260 \mathrm{~kJ} / \mathrm{kg}$ )

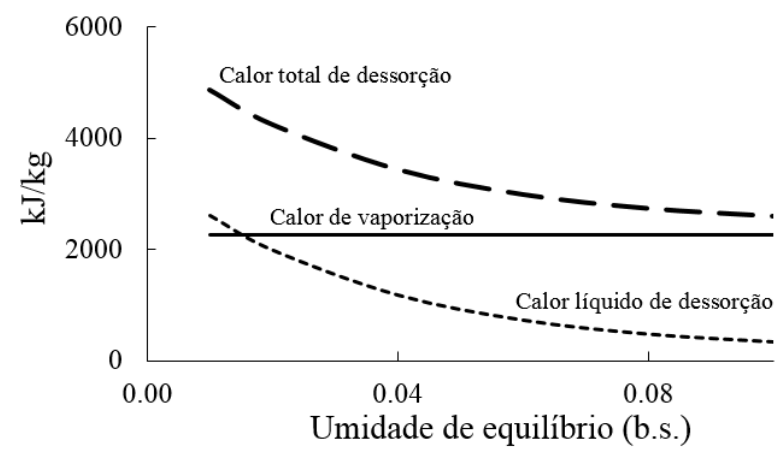

O calor total de dessorção calculado variou de 4864 a $2591 \mathrm{~kJ} / \mathrm{kg}$, respectivamente para umidades de 0,01 e 0,1 (b.s.), de modo que a quantidade de energia requerida para remover uma unidade de massa de água durante a secagem aumentou com a redução do conteúdo de umidade. Os resultados obtidos estão de acordo com aqueles dos pesquisadores Majd et al (2013), que determinaram o calor líquido isostérico de sementes de uva cultivar Seiahe-Shiraz, obtendo valores aproximados de $3600 \mathrm{~kJ} / \mathrm{kg}$, para umidade próxima a 0,2 (b.s.). Demais trabalhos que estudaram o calor isostérico de grãos, como arroz (TOGRUL e ARSLAN, 2006); feijão de corda (AJIBOLA et al. 2003); soja (AVIARA et al. 2004); nozes (TOGRUL e ARSLAN, 2007); e gergelim (AVIARA et al. 2002), chegaram a valores próximos aos determinados no presente estudo.

Observou-se que pequenos acréscimos na umidade dos grãos acarretaram grandes decréscimos no calor de dessorção. Esse fato pode ser explicado pela existência de maior quantidade de água não ligada quando os teores de umidade são maiores. A medida que a umidade diminui, diminui também a quantidade de água livre, portanto a energia necessária para a evaporação aumenta. A quantidade de energia requerida passa a ser, não somente aquele necessária à evaporação simples, mas também à energia para quebrar as ligações água-estrutura sólida.

A entropia diferencial foi calculada a partir dos valores do calor total de dessorção, com emprego da Equação 5, o efeito da umidade de equilíbrio na entropia diferencial é ilustrado na Figura 3. A entropia diferencial foi ajustada, em função da umidade, conforme modelo exponencial, Equação 13, apresentando $\mathrm{R}^{2}=0,996$.

$\mathrm{S}_{\mathrm{D}}=0,0984 \mathrm{X}_{\mathrm{E}}^{-0,87}$

Figura 3 - Efeito da umidade de equilíbrio na entropia diferencial

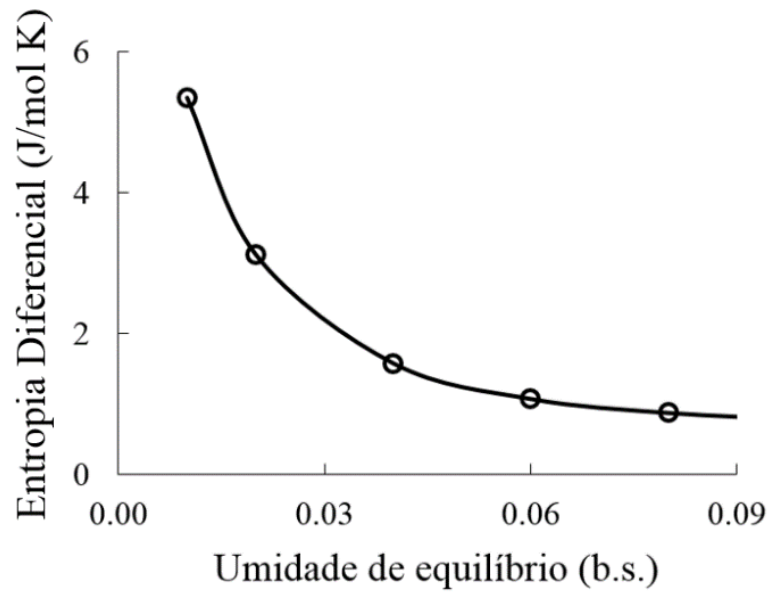

Verifica-se na Figura 3, que a entropia diferencial cai drasticamente com o aumento da umidade, conforme também observado na secagem de grãos de arroz (TOGRUL e ARSLAN, 2006). A entropia diferencial apresentou valor máximo de 5,34 e mínimo de $0,77 \mathrm{~J} / \mathrm{mol} \mathrm{K}$, respectivamente para 0,01 e 0,1 (b.s.).

Para o cálculo da pressão de espalhamento, os dados de equilíbrio de Menezes (2014), foram ajustados ao modelo de 
BET, Equação 9, sendo obtidos os valores respectivos dos parâmetros $\mathrm{A}$ e $\mathrm{B}$, iguais a 555364 e 0,0266 para $25^{\circ} \mathrm{C} ; 64,6889$ e 0,0283 para $35{ }^{\circ} \mathrm{C}$; e 4,9300 e 0,0321 para $50{ }^{\circ} \mathrm{C}$. Os valores de $\mathrm{R}^{2}$ calculados foram 0,$84 ; 0,90 \mathrm{e}$ 0,98 .

A relação entre a pressão de espalhamento e a atividade da água foi analisada nas temperaturas de 25,35 e $50{ }^{\circ} \mathrm{C}$, sendo a entalpia integral determinada pela Equação 10. A Figura 4 apresenta o comportamento da pressão de espalhamento em função da atividade da água.

Figura 4 - Variação da pressão de espalhamento em função da atividade da água

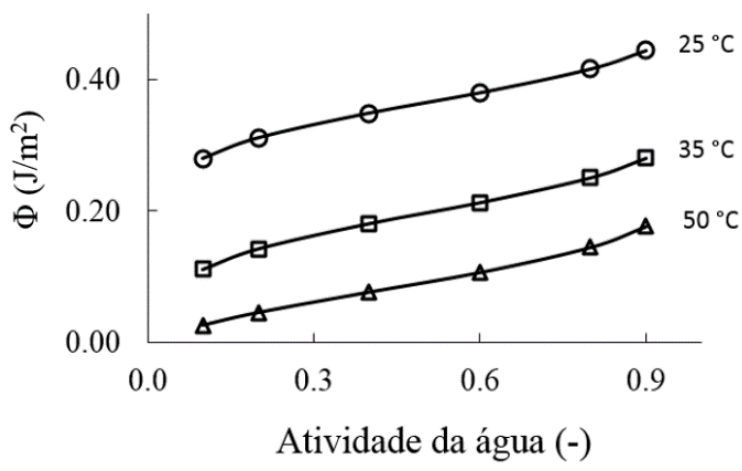

Observa-se que os valores da pressão de espalhamento aumentam discretamente com o aumento da atividade da água, sendo que com o aumento da temperatura, os valores são reduzidos. A mesma tendência foi observada na literatura, para a secagem de outros grãos: arroz (TOGRUL e ARSLAN, 2006), feijão de corda (AJIBOLA et al. 2003), soja (Aviara et al. 2004), nozes (TOGRUL e ARSLAN, 2007) e gergelim (AVIARA et al. 2002), sendo que a mesma ordem de grandeza foi calculada por Majd et al. (2013), para sementes de uva cultivar Seiahe-Shiraz, nas temperaturas de 35 a $65{ }^{\circ} \mathrm{C}$.

Para estimar a entalpia integral, foi empregada a Equação 6, sendo que tanto a entalpia quanto a entropia integral foram calculadas em pressão de espalhamento constante, e para cada temperatura foram consideradas quatro teores de umidade, As relações entre a entalpia e a entropia integral com a umidade são ilustradas na Figura 5 e 6.

Figura 5 - Variação da entalpia integral em função da umidade

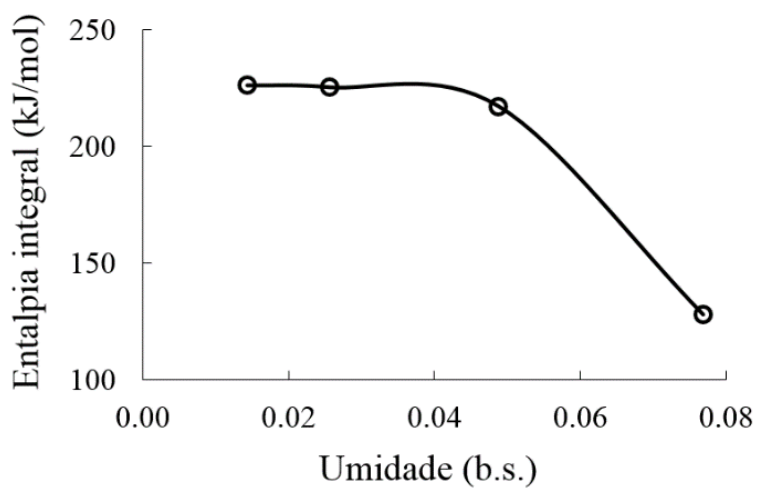

Figura 6 - Variação da entropia integral em função da umidade

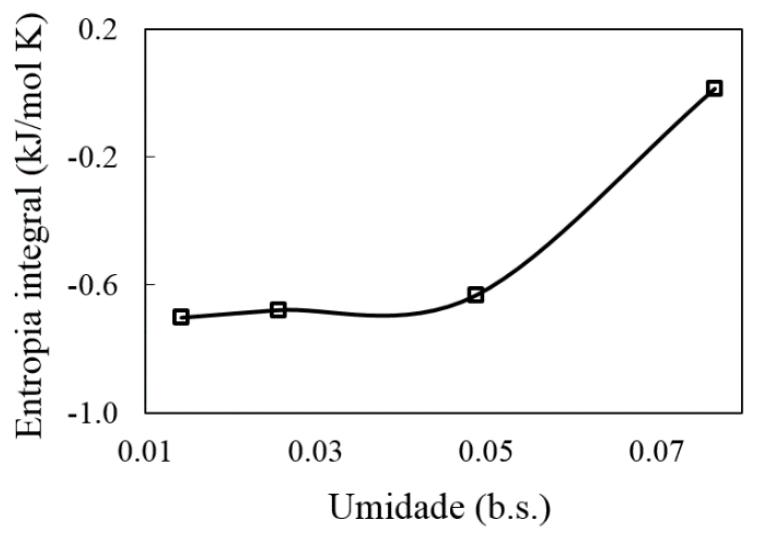

A entalpia integral permanece aproximadamente constante, $220 \mathrm{~kJ} / \mathrm{mol}$, entre os teores de umidade de 0,01 e 0,05, sofrendo decréscimo acentuado a partir daí, atingindo valore de $128 \mathrm{~kJ} / \mathrm{mol}$ próximo à 0,08 (b.s.). Valores próximos foram encontrados para grãos de feijão de corda (AJIBOLA et al. 2003) e soja (AVIARA et al. 2004).

De acordo com Aviara et al. (2004), este comportamento pode ser devido ao fato de que, com o aumento da umidade das sementes, os sitos de sorção saturam e as moléculas de água são mantidas junto às partículas mais devido à tensão superficial que pela adsorção química. Quanto mais fracas são as forças de tensão 
superficial, mais facilmente são quebradas, resultando em menor entalpia integral.

Por sua vez, a entropia integral aumenta com o acréscimo do teor de umidade, variando ente aproximadamente - 0,702 a $0,013 \mathrm{~kJ} / \mathrm{mol} \mathrm{K}$, entre 0,01 e 0,07 (b.s.). De acordo com Togrul e Arslsn (2007), uma queda na entropia integral indicaria perda de liberdade ou aleatoriedade das moléculas de água, à medida que os sítios tornam-se saturados. Já, segundo os autores, o aumento da entropia integral reflete a ocorrência de ligações realizadas mais livremente e formação de multicamadas.

\section{CONCLUSÕES}

Foram determinadas as funções termodinâmicas diferenciais e integrais (entalpia e entropia), a partir das isotermas de dessorção de grãos de uva Vitis vinífera.

O calor total de dessorção calculado variou entre 4864 e $2591 \mathrm{~kJ} / \mathrm{kg}$, análogo a dados da literatura, sendo que a quantidade de energia requerida para dessorção aumentou com a redução do conteúdo de umidade. A entropia diferencial caiu drasticamente com o aumento da umidade, conforme literatura, apresentando valor máximo de 5,34 e mínimo de $0,77 \mathrm{~J} / \mathrm{mol} \mathrm{K}$. A pressão de espalhamento, analisada nas temperaturas de 25,35 e $50{ }^{\circ} \mathrm{C}$, aumentou discretamente com o aumento da atividade da água, sendo que com o aumento da temperatura, os valores foram reduzidos, a mesma tendência foi observada na literatura para outras sementes. A entalpia integral permaneceu aproximadamente constante, $220 \mathrm{~kJ} / \mathrm{mol}$, sofrendo decréscimo acentuado a partir de 0,05 (b.s.), valores próximos aos encontrados na literatura. A entropia integral aumentou com o acréscimo do teor de umidade, variando ente aproximadamente - 0,702 a $0,013 \mathrm{~kJ} / \mathrm{mol} \mathrm{K}$, entre 0,01 e 0,07 (b.s.).
A Parâmetro do modelo

$\mathrm{A}_{\mathrm{m}} \quad$ Área da molécula de água $\left(\mathrm{m}^{2}\right)$

$\mathrm{a}_{\mathrm{w}} \quad$ Atividade da água (-)

$\mathrm{a}_{\mathrm{w}}{ }^{*}$ Média geométrica da atividade da água (-)

B Parâmetro do modelo

b.s. Base seca $(\mathrm{kg} / \mathrm{kg})$

D Parâmetro do modelo

$\mathrm{H}_{\mathrm{I}} \quad$ Entalpia integral líquida $(\mathrm{kJ} / \mathrm{kg})$

$\mathrm{K}_{\mathrm{B}} \quad$ Constante de Boltzmann's (J/K)

$\mathrm{P}_{\mathrm{P}} \quad$ Pressão parcial $(\mathrm{Pa})$

$\mathrm{P}_{\mathrm{V}} \quad$ Pressão de vapor $(\mathrm{Pa})$

$\mathrm{R}$ Constante dos gases ideais $(\mathrm{J} / \mathrm{mol} \mathrm{K})$

$\mathrm{S}_{\mathrm{D}} \quad$ Entropia diferencial $(\mathrm{kJ} / \mathrm{kg} \mathrm{K})$

$\mathrm{S}_{\mathrm{I}} \quad$ Entropia integral líquida $(\mathrm{kJ} / \mathrm{mol} \mathrm{K})$

$\mathrm{T}$ Temperatura $\mathrm{T}\left({ }^{\circ} \mathrm{C}\right)$

$\mathrm{X}_{\mathrm{E}} \quad$ Umidade de equilíbrio (b.s.)

$\mathrm{X}_{\mathrm{m}} \quad$ Umidade da monocamada (b.s.)

$\phi \quad$ Pressão de espalhamento $\left(\mathrm{J} / \mathrm{m}^{2}\right)$

$\lambda_{\mathrm{D}} \quad$ Calor total de dessorção $(\mathrm{kJ} / \mathrm{kg})$

$\lambda_{\mathrm{N}}$ Calor líquido isostérico de dessorção $(\mathrm{kJ} / \mathrm{kg})$

$\lambda_{\mathrm{V}} \quad$ Calor latente de vaporização da água pura $(\mathrm{kJ} / \mathrm{kg})$

\section{REFERÊNCIAS}

AJIBOLA, O. O., AVIARA, N. A. AJETUMOBI, O. E. Sorption equilibrium and thermodynamic properties of cowpea (Vigna unguiculata). Journal of Food Engineering, v.58, p.317-324, 2003.

ALONSO, D. M. J. Modeling of grain dryers: thin layer to deep beds. 2011. Dissertação (Mestrado em Engenharia de Bioprocessos) - McGill University - Montreal, 2011.

AVIARA, N. A., AJIBOLA, O. O., DAIRO, U. O. Thermodynamics of Moisture Sorption in Sesame Seed. Biosystems Engineering, v.83, p.423-431, 2002.

\section{NOMENCLATURA}


AVIARA, N. A., AJIBOLA, O. O., ONI, S. A. Sorption Equilibrium and Thermodynamic Characteristics of Soya Bean, Biosystems Engineering, v.87, p.179-190, 2004.

BECKER, H. A., SALLANS, H. R. A. Study of the desorption isotherms of wheat at $5{ }^{\circ} \mathrm{C}$ and $50{ }^{\circ} \mathrm{C}$. Cereal Chemistry, v.33, p.79-91, 1956.

BUSTAMANTE, M. A., MORAL, R., PAREDES, C., PÉREZ-ESPINOSA, A., MORENOCASELLES, J., PÉREZ-MURCIA, M. D. Agrochemical characterization of the solid byproducts and residues from the winery and distillery industry. Waste Manage, v.28, p.372-380, 2008.

CAURIE, M. Derivation of full range moisture sorption isotherms. In: ROCKLAND, L. B., STEWART, G. F. Water activity: influences on food quality. New York: Academic Press, 1981.

DAVIDOV-PARDO, G., MCCLEMENTS, D. J. Nutraceutical delivery systems: Resveratrol encapsulation in grape seed oil nanoemulsions formed by spontaneous emulsification. Food Chemistry, v.167, p.205-212, 2015.

DENT, R. W. A multilayer theory for gas sorption. Part I: Sorption of a singlegas. Textile Research Journal, v.47, p.145-152, 1977.

FADHEL, A., KOOLI, S., FARHAT, A., BELLGHITH, A. Study of the solar drying of grapes by three different processes. Desalination, v.185, p.535-541, 2005.

FASINA, O. O., AJIBOLA, O. O., TYLER, R. $\mathrm{T}$. Thermodynamics of moisture sorption in winged bean seed and gari. Journal of Food Process Engineering, v.22, p.405-418, 1999.
FLUCK, R. C., BAIRD, C. D. Energy requirements for agricultural inputs. In: Agricultural Energetics. AVI Publishing Company, Inc., Westport, Connecticut, 1980.

GAL, S. Recent advances in techniques for the determination of sorption isotherms. In: Water Relations of Foods (Duckworth R B, ed), Academic Press, London, 1965.

GUINÉ, R. P. F., HENRRIQUES, F., BARROCA, M. J. Mass Transfer Coefficients for the Drying of Pumpkin (Cucurbita moschata) and Dried Product Quality. Food Bioprocess Technology, v.5, p.176-183, 2012.

GUNASEKARAN, S., THOMPSON, T. L. Optimal energy management in grain drying. Critical Reviews in Food Science and Nutrition, v.25, p.1-48, 1986.

HENDERSON, S. M. A basic concept of equilibrium moisture. Agriculture Engineering, v.33, p.29-32, 1952.

MADAMHA, P. S., DRISCOLL, R. H., BUCKLE, K. A. Enthalpy-entropy compensation models for sorption and browning of garlic. Journal of Food Engineering, v.28, p.109-119, 1996.

MAJD, K. M., KARPARVARFARD, S. H., FARAHNAKY, A., JAFARPOUR, K. Thermodynamic of Water Sorption of Grape Seed: Temperature Effect of Sorption Isotherms and Thermodynamic Characteristics. Food Biophysics, v.8, p.1-11, 2013.

MAZZA, G., LEMAGUER, M. Water sorption properties of yellow globe onion. Journal of the Canadian Institute of Food Science and Technology, v.11, p. 189-193, 1978. 
MCMinN, W. A. M., MAGEE, T. R. A. Thermodynamic properties of moisture sorption of potato. Journal of Food Engineering, v.60, p.157-165, 2003.

MENEZES, M. L. Estudo dos processos de secagem e extração para produção de óleo bruto de semente de uva. 2014. Tese (Doutorado em Engenharia Química) Universidade Estadual de Maringá, Maringá, 2014.

OTHMER, D. Correlating vapor pressure and latente heat data. Industry Engineering Chemistry, v.32, p.841-856, 1940.

OUERTANI, S., AZZOUZ, S., HASSINI, L. KOUBAA, A., BELGHITH, A. Moisture sorption isotherms and thermodynamic properties of Jackpine and palm wood: Comparative study. Industrial Crops and Products, v.56, p.200-210, 2014.

PEDRO, M. A. M., TELIS-ROMERO, J., TELIS, V. R. N. Effect of drying method on the adsorption isotherms and isosteric heat of passion fruit pulp poder. Ciência e Tecnologia de Alimentos, v.30, p.993-1000, 2010.

PERUSSELLO, C. A., KUMAR, C., CASTILHOS, F., KARIM, M. A. Heat and mass transfer modeling of the osmoconvective drying of yacon roots (Smallanthus sonchifolius). Applied Thermal Engineering, v.63, p.23-32, 2014.

RAGHAVAN, V. G. S., SOSLE, V. Grain Drying, IN Handbook of industrial drying. Boca Raton, FL: CRC/Taylor \& Francis, 2007.

SILVA, M. M., GOUVEIA, J. P. G., ALMEIDA, F. A. C., SILVA, M. M. Demanda energética envolvida no processo de dessorção de umidade em polpa de manga. Revista Brasileira de Produtos Agroindustriais, v.4, p.107-117, 2002.
STAMM, A. J. Wood and cellulose science. New York: The Ronald Press, 1964.

STURM, B., VEGA, A. M. N., HOFACKER, W. C. Influence of process control strategies on drying kinetics, color and shrinkage of air dried apples. Applied Thermal Engineering, v. 62, p.455-460, 2014.

TOGRUL, H., ARSLAN, N. Moisture Sorption Behaviour and Thermodynamic Characteristics of Rice stored in a Chamber under Controlled Humidity. Biosystems Engineering, v.95, p.181-195, 2006.

TOGRUL, H., ARSLAN, N. Moisture sorption isotherms and thermodynamic properties of walnut kernels. Journal of Stored Products Research, v.43, p.252-264, 2007.

TOLABA, M. P., PELTZER, M., ENRI'QUEZ, N., POLLIO, M. A. Grain sorption equilibria of quinoa grains. Journal of Food Engineering, v.61, p.365-371, 2004.

VERNA, L. R. New methods for on-the-farm rice drying: solar and biomass. In: Wayne, E.M., Wadsworth, J.I. (Eds.), Rice Science and Technology. Marcel Dekker, Inc., 270 Madison Avenue, New York, USA, 1994.

\section{AGRADECIMENTO}

Os autores agradecem a Coordenação de Aperfeiçoamento de Pessoal de Nível Superior (CAPES) pela concessão da bolsa de doutorado à aluna Gracielle Johann. 